\title{
Terahertz scanning imaging with a subwavelength plastic fiber
}

\author{
Ja-Yu Lu, Chui-Min Chiu, Chung-Chiu Kuo, Chih-Hsien Lai, and Hung-Chung Chang \\ Department of Electrical Engineering and Graduate Institute of Photonics and Optoelectronics, \\ National Taiwan University, Taipei 10617, Taiwan \\ Yuh-Jing Hwang \\ Institute of Astronomy and Astrophysics, Academia Sinica, Taipei 10617, Taiwan \\ Ci-Ling Pan \\ Graduate Institute of Electro-Optical Engineering, National Chiao-Tung University, Hsinchu 30056, Taiwan \\ Chi-Kuang Sun ${ }^{\text {a) }}$ \\ Department of Electrical Engineering and Graduate Institute of Photonics and Optoelectronics, \\ National Taiwan University, Taipei 10617, Taiwan and Research Center for Applied Sciences, \\ Academia Sinica, Taipei 10617, Taiwan
}

(Received 18 July 2007; accepted 1 November 2007; published online 26 February 2008)

\begin{abstract}
The feasibility to perform fiber-scanning terahertz imaging utilizing a terahertz subwavelength plastic fiber is investigated. Our study shows that, with a low $(<1 \%)$ fractional power inside the fiber core, the bending loss of the terahertz subwavelength fiber is acceptable to enable large area scanning without seriously sacrificing the signal-to-noise ratio of the acquired image. With a transmission geometry, this feasibility is demonstrated by direct two-dimensional scanning of the terahertz subwavelength fiber output end to image different biological samples and concealed substances. () 2008 American Institute of Physics. [DOI: 10.1063/1.2816122]
\end{abstract}

Recently, various kinds of terahertz waveguides or fibers have been developed for efficient transmission of terahertz waves, including hollow core metal waveguides, ${ }^{1}$ bare metal wires, ${ }^{2}$ parallel plate metal waveguides, ${ }^{3}$ plastic ribbon waveguides, ${ }^{4}$ photonic crystal fibers, ${ }^{5}$ Bragg fibers, ${ }^{6}$ and plastic subwavelength fibers. ${ }^{7,8}$ Some of them have an improved attenuation constant ${ }^{1-8}\left(<0.01 \mathrm{~cm}^{-1}\right)$ and high coupling efficiency, ${ }^{7,8}$ and could transmit terahertz waves with less dispersion or even dispersion-free. ${ }^{2,3}$ However, applications based on the demonstrated terahertz waveguides are still rare. At the optical frequency, optical fibers have been applied to various fields such as communication, wavelength generation, and dispersion control, among which the most attractive application is the fiber-based imaging ${ }^{9,10}$ that enables in vivo imaging or sensing in a less-invasive manner and has been extensively applied in endoscopy ${ }^{9}$ and microscopy, ${ }^{10}$ test of drugs, and explosive detections. ${ }^{11}$ For biomedical applications, many biomolecules have their characteristic absorption spectra in the terahertz frequency range, enabling marker-free and direct recognition of molecular distribution based on their distinct terahertz fingerprints. Therefore, if terahertz fiber scanning imaging could be realized, it will not only allow remote coupling to a bulky terahertz generation setup from the detected sample and allow flexible terahertz wave guidance, but will also greatly expand terahertz technology to intravital molecular imaging just like its optical counterpart. Recently we have demonstrated a terahertz subwavelength plastic fiber ${ }^{7,8}$ with a low attenuation constant $\left(<0.01 \mathrm{~cm}^{-1}\right)$ and a high free-space direct-coupling efficiency $(>20 \%)$. For fiber-scanning imaging applications, terahertz fiber is required not only to be with a low attenuation and a high coupling efficiency for high signal-to-noise ratio (SNR), but with a low bending loss for large area scan-

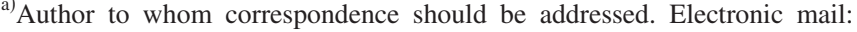
sun@cc.ee.ntu.edu.tw. ning. However, the demonstrated terahertz subwavelength plastic fiber has a loosely confined terahertz field (typical $<1 \%$ terahertz power inside the fiber core), which could be sensitive to fiber bending. In this letter, we report our study on the feasibility of adopting the terahertz subwavelength fiber for terahertz fiber scanning imaging. We investigated the bending loss characteristic of the subwavelength plastic fiber under the low-fractional-power-in-the-core regime, and further utilized it to construct a transmission terahertz fiber scanning imaging system. Through acquiring fiber-scanned terahertz images, this feasibility study shows that the lowloss subwavelength terahertz fiber has the capability for large area image scanning with a high SNR and could be promising for various fiber scanning applications.

The adopted subwavelength polyethylene (PE) fiber was made by a melt-spinning method ${ }^{8}$ with a minimum attenuation constant typically on the order of $0.01 \mathrm{~cm}^{-1}$. Two PE films with a hole punched at the center and attached on $1 \mathrm{in}$. metal rings were used for tightly mounting the two ends of the PE fiber. A terahertz power transmission measurement through a $180-\mu \mathrm{m}$-thick fiber was performed similar to a previous report, ${ }^{7}$ showing that the subwavelength fiber has a minimum attenuation constant around $380 \mathrm{GHz}{ }^{8}$ We then conducted the fiber-bending loss measurement with the 180- $\mu \mathrm{m}$-diameter fiber at $380 \mathrm{GHz}$. A cw Gunn oscillator module ${ }^{12,13}$ with a size of $10 \times 5 \times 15 \mathrm{~cm}^{3}$ was used as the bending loss measurement source. The signal from the Gunn oscillator $^{13}$ passed through a frequency multiplier and fed into a horn antenna for $\mathrm{cw}$ terahertz wave radiation. The adopted Gunn oscillator module provided $\mathrm{cw}$ terahertz power at $380 \mathrm{GHz}$ around $220 \mu \mathrm{W}$. The free-running terahertz waves, collected and focused by a pair of parabolic mirrors, were coupled into the subwavelength plastic fiber. With a fractional-power-inside-the-core $\eta$ as low as $0.2 \%$, attenuation constant of $0.003 \mathrm{~cm}^{-1}$ at $380 \mathrm{GHz}$ can be extracted, by comparing the measured terahertz output powers after propa- 


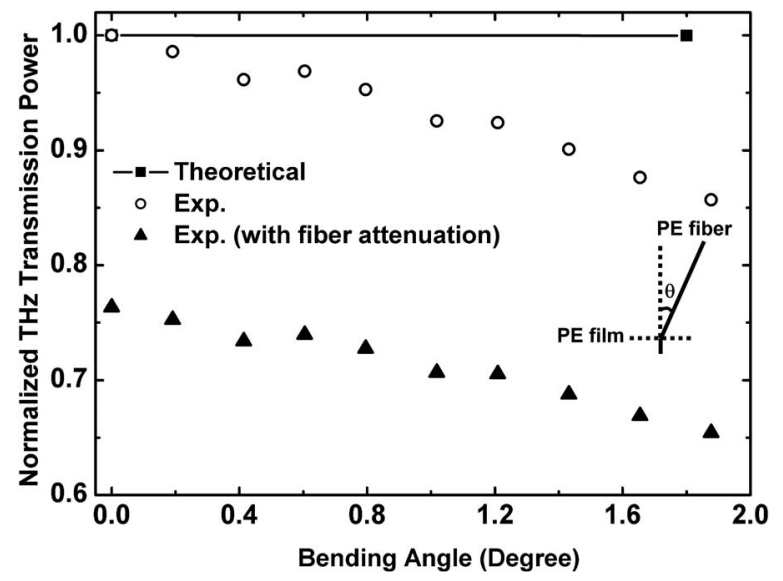

FIG. 1. The measured normalized $380 \mathrm{GHz}$ terahertz power transmission ratio at different fiber bending angles (open circles). The solid squares and the solid line are the theoretical results based on the FD-BPM (Ref. 14) simulation. The solid triangles are the terahertz power transmission ratio including fiber attenuation. Inset shows the configuration for the bending loss measurement.

gating through different fiber lengths. ${ }^{8}$ By holding the fiber straight ${ }^{8}$ and by laterally moving the output end of the fiber, Fig. 1 shows the measured terahertz transmitted power of the 180- $\mu \mathrm{m}$-diameter PE fibers as a function of bending angle $\theta$, after normalization respected to the zero degree. Our measurement shows that the subwavelength fiber is more sensitive to the bending loss than a simple theoretical prediction (solid line). A simple theoretical estimation of the bending loss at the abrupt junction between two connected straight fibers with a bending angle $\theta$ could be based on the mode overlap integral of the two fiber modes at the junction, which is simply $\cos \theta$. For $\theta$ smaller than $1.8^{\circ}$, the loss would be $1-\cos \theta \sim 10^{-4}$, which is negligibly small and is consistent with the simulated result based on a home-established threedimensional Yee-mesh-based full-vectorial finite-difference beam propagation method ${ }^{14}$ (FD-BPM). Based on the FD-BPM $^{14}$ simulation, negligibly small terahertz loss was observed at a small bending angle of $1.8^{\circ}$, even less than our experimental observations. The experimentally measured bending loss was also found to decrease when the $\eta$ increases, accompanied with a higher attenuation constant. ${ }^{7}$ For example, at a bending angle of $2^{\circ}$, the experimentally measured transmitted terahertz power (versus $0^{\circ}$ ) after normalization is 0.91 for a fiber with a $\eta$ of $3.6 \%$, but only 0.86 for a fiber with a $\eta$ of $0.2 \%$. However, the fiber attenuation constant for the specific fiber at its operation wavelength with a $\eta$ of $3.6 \%$ was measured to be $0.011 \mathrm{~cm}^{-1}$. By considering the total fiber transmission after a $90 \mathrm{~cm}$ long fiber, including bending loss at $2^{\circ}$ and fiber attenuation loss, total transmission of 0.65 versus 0.34 can be found for $\eta$ of $0.2 \%$ versus $3.6 \%$. Our study reveals the fact that even with a higher bending loss, the low $\eta$ fiber is preferred for the scanning applications. Our study indicates that the total transmission loss in the $180-\mu \mathrm{m}$-diameter fiber is acceptable under the loosely terahertz waveguiding regime $(\eta \sim 0.2 \%)$ for scanning a $6 \times 6 \mathrm{~cm}^{2}$ area with a $90 \mathrm{~cm}$ fiber length.

To further investigate the feasibility to perform fiberscanning terahertz imaging by utilizing the low-loss subwavelength plastic fiber, we integrated the plastic fiber with the cw Gunn oscillator and a Golay cell ${ }^{15}$ to construct a terahertz fiber scanning imaging system for imaging spatially Downloaded 16 Feb 2009 to 140.112 .113 .225 . Redistribution subje

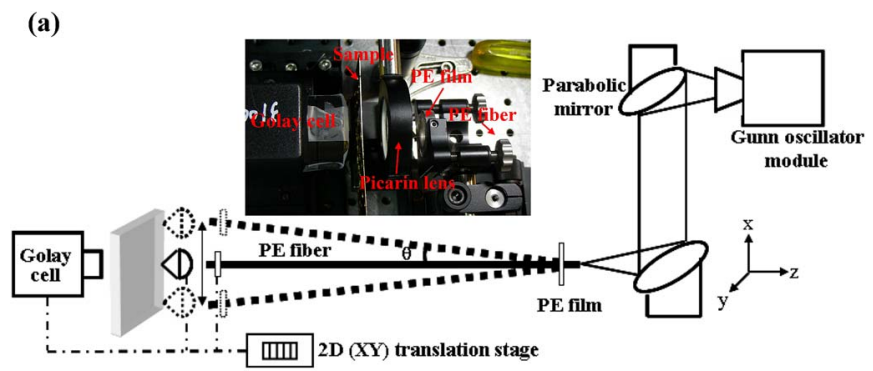

(b)

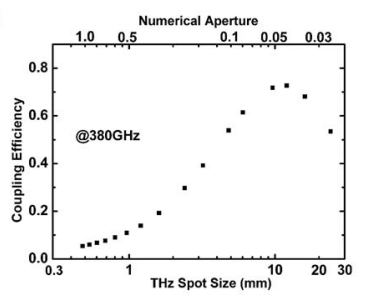

(c)

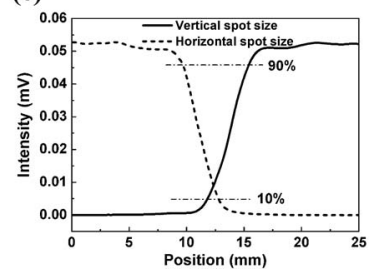

FIG. 2. (Color online) Schematic diagram of the testing terahertz fiber scanning imaging system with a transmission geometry. The photo shows the detailed scanning head. (b) The simulated coupling efficiency. (c) Terahertz spot size measurement after the picarin lens. Solid (dashed) line is the terahertz transmission power versus the position of sharp knife edge measured in the vertical (horizontal) direction. Measured vertical and horizontal diameters were 3.6 and $3.1 \mathrm{~mm}$, respectively.

fixed samples. Figure 2(a) shows the schematic diagram and top view of the fiber scanning imaging system. The $\mathrm{cw}$ $380 \mathrm{GHz}$ waves from the Gunn oscillator were collected and focused by two parabolic mirrors to couple into the $180-\mu \mathrm{m}$-diameter subwavelength plastic fiber. We used the PE wire with a length of $90 \mathrm{~cm}$, same as the experiment in Fig. 1. Figure 2(b) shows the simulated coupling efficiency of a 180- $\mu \mathrm{m}$-thick PE wire versus different focused terahertz Gaussian spot size after a lens, which is the overlap integral of the fiber mode and the input terahertz field. Agreeing with the simulated result, with a measured $4.2 \mathrm{~mm}$ focused terahertz spot size after two parabolic mirrors, we experimentally achieved a free-space fiber coupling efficiency over $50 \%$. The cw terahertz output after the fiber was focused by a terahertz lens (picarin lens, numeric aperture $\sim 0.42$ ) to a stationary sample. A room-temperature operated Golay cell ${ }^{12}$ was used for measuring the terahertz transmission power after the sample. The terahertz transmission image was accomplished by two-dimensional (2D) scanning of the output end of the PE fiber, together with the picarin lens and Golay cell $^{15}$ which were all mounted on a motorized stage. The system has a $26 \%$ total terahertz power transmission rate, including the fiber input-coupling loss, bending loss (less than $2^{\circ}$, corresponding to a scanning area of $6 \times 6 \mathrm{~cm}^{2}$ if fiber was $90 \mathrm{~cm}$ long), fiber attenuation, and the picarin lens inserting loss. A lock-in amplifier and a $20 \mathrm{~Hz}$ chopper were used to increase SNR. With a $100 \mathrm{~ms}$ integration time, the SNR of the imaging system can be better than 3000:1. Figure 2(c) shows the measurement of the focused terahertz spot size after the picarin lens, by a knife-edge method. The measured spot size was determined by the $90 \%$ and $10 \%$ transmissions after a sharp knife edge. The measured focus spot size was on the order of $3 \mathrm{~mm}$ and was close to a circular transverse mode, which is also close to the theoretical diffraction limit $(2.7 \mathrm{~mm})$.

Figure 3 shows some examples of the acquired $380 \mathrm{GHz}$ terahertz images, wherein Figs. 3(a)-3(d) show images of a metal pattern $\left(8 \times 6.5 \mathrm{~cm}^{2}\right)$, a matchbox $\left(5 \times 3.5 \mathrm{~cm}^{2}\right)$, a dry to AIP license or copyright; see http://apl.aip.org/apl/copyright.jsp 


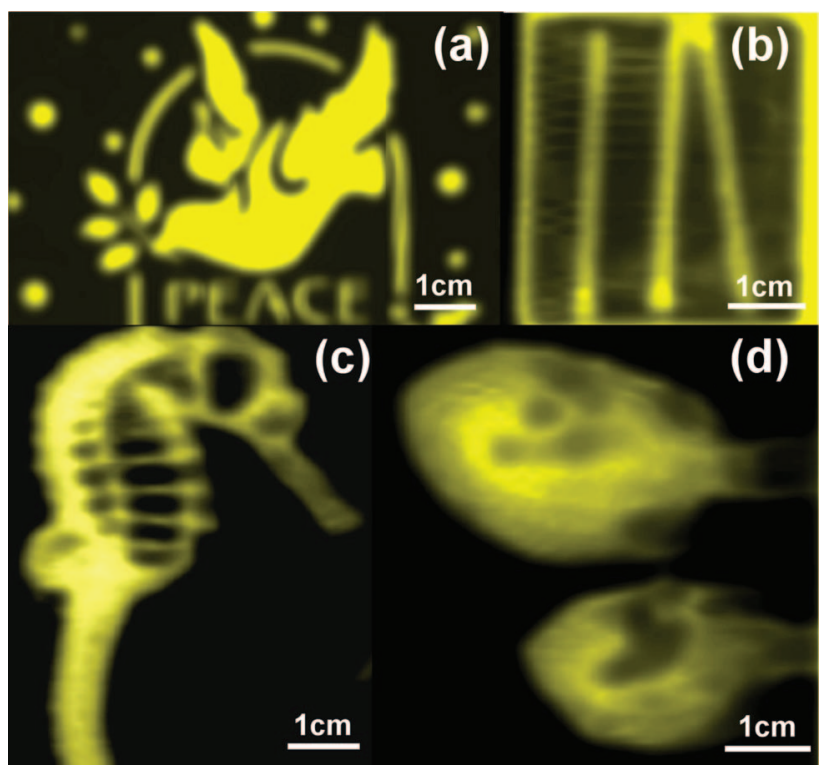

FIG. 3. (Color) Fiber-scanning terahertz images of (a) a metal pattern with a word "peace" and a dove figure, (b) a paper-made matchbox with three matches inside, (c) a dry seahorse, and (d) two dry fishes. In (a), the yellow region is with high terahertz transmission while the dark region is with low terahertz transmission. In (b)-(d), the yellow region is with low terahertz transmission while the dark region is with high terahertz transmission.

seahorse $\left(8.5 \times 4 \mathrm{~cm}^{2}\right)$, and two dry fishes $\left(5 \times 6 \mathrm{~cm}^{2}\right)$. They were original terahertz images, with a scanned step size of $0.5 \times 0.5 \mathrm{~mm}^{2}$, that were not normalized to the background, that is, we did not correct the angle-dependent bending loss. To prevent the instability of our adopted Golay cell due to vibration, the integration time in this preliminary experiment was set to be $300 \mathrm{~ms}$, and the acquisition time of a terahertz image with a size of $5 \times 6 \mathrm{~cm}^{2}(100 \times 120$ pixels $)$ was about $1 \mathrm{~h}$. However, with the state-of-the-art response time of a Golay cell $<20 \mu$ s, a Golay-cell-based fiber scanning imaging system could be potentially with a scanning rate higher than $5 \mathrm{frame} / \mathrm{s}$. Also our results indicate that the transmission power dropped to $\sim 80 \%$ of the peak value with $2^{\circ}$ fiber bending. Take advantage of a 90-cm-long PE fiber, the SNR of a terahertz image with a size of $10 \times 10 \mathrm{~cm}^{2}$, thus, still exceeded 2200:1 at the maximum bending region. These results reveal that the terahertz fiber-scanning imaging system is able to scan over a large area of metal materials, biological specimens, and concealed objects. In Fig. 3(b), terahertz waves transmitted through a paper-made box and identified three matches hidden inside. Figures 3(c) and 3(d) are terahertz transmission images of biological samples, where the dark region represents high terahertz transmission and the yellow region is with low transmission. We could distinguish different structures inside the dry seahorse including spine, brain cavity, abdominal cavity, and somite. In Fig. 3(d), there are more low-transmission regions compared to Fig. 3(c), because the body of a fish is denser than that of a seahorse. However, we could also see through the thoracic cavity inside the fish body, where the structure is looser.

In conclusion, we studied the feasibility to perform fiberscanning terahertz imaging by utilizing a low-loss terahertz subwavelength plastic fiber. Our study shows that, with a low $(<1 \%)$ fractional power inside the core and a long fiber, the bending loss of the subwavelength fiber is acceptable to enable large area scanning without seriously sacrificing the SNR of the acquired image. With a transmission detection geometry, this feasibility is further demonstrated by direct 2D scanning of the terahertz subwavelength fiber output end to image different biological samples and concealed substances. Our study indicates that the terahertz subwavelength fiber would be a good candidate for future development of terahertz endoscopes and is suitable for fiber-scanning, remote sensing, and security applications.

This work was sponsored by the National Science Council of Taiwan under Grant Nos. 96-2628-E-002-043-MY3 and 96-2120-M-002-014, Frontier Research of NTU (95R0110), the Program for Promoting Academic Excellence of University Project in NCTU, National Health Research Institute of Taiwan (NHRI-EX96-9201EI), and NTU Research Center For Medical Excellence.

${ }^{1}$ J. Harrington, R. George, P. Pedersen, and E. Mueller, Opt. Express 21, 5263 (2004).

${ }^{2}$ K. Wang and D. M. Mittleman, Nature 432, 376 (2004).

${ }^{3}$ R. Mendis and D. Grischkowsky, Opt. Lett. 26, 846 (2001).

${ }^{4}$ R. Mendis and D. Grischkowsky, J. Appl. Phys. 88, 4449 (2000).

${ }^{5}$ H. Han, H. Park, M. Cho, and J. Kim, Appl. Phys. Lett. 80, 2634 (2002).

${ }^{6}$ M. Skorobogatiy and A. Dupuis, Appl. Phys. Lett. 90, 113514 (2007).

${ }^{7}$ L.-J. Chen, H.-W. Chen, T.-F. Kao, J.-Y. Lu, and C.-K. Sun, Opt. Lett. 31, 308 (2006).

${ }^{8}$ H.-W. Chen, Y.-T. Li, J.-L. Kuo, J.-Y. Lu, L.-J. Chen, C.-L. Pan, and C.-K. Sun, Opt. Lett. 32, 1017 (2007).

${ }^{9}$ A. L. Polglase, W. J. McLaren, S. A. Skinner, R. Kiesslich, M. F. Neurath, and P. M. Delaney, Gastrointest. Endosc. 62, 686 (2005).

${ }^{10}$ F. Helmchen, M. S. Fee, D. W. Tank, and W. Denk, Neuron 31, 903 (2001).

${ }^{11}$ B. A. Flusberg, E. D. Cocker, W. Piyawattanametha, J. C. Jung, E. L. M. Cheung, and M. J. Schnitzer, Nat. Methods 2, 941 (2005).

${ }^{12}$ T. W. Crowe, W. L. Bishop, D. W. Porterfield, J. L. Hesler, and R. M. Weikle, IEEE J. Solid-State Circuits 40, 2104 (2005).

${ }^{13}$ J. E. Carlstrom, R. L. Plambeck, and D. D. Thornton, IEEE Trans. Microwave Theory Tech. 33, 610 (1985).

${ }^{14}$ J. Yamauchi, T. Mugita, and H. Nakano, J. Lightwave Technol. 23, 1947 (2005).

${ }^{15}$ Golay cells, Microtech Instruments, Inc., Eugene, Oregon (see www.mtinstruments.com). 